\title{
Numerical and experimental investigation on a maritime radar scanner
}

\author{
S. Muggiasca, F. Ripamonti, D. Rocchi \& A. Zasso \\ Mechanical Engineering Department, Politecnico di Milano, \\ Milano, Italy
}

\begin{abstract}
This paper presents the study of the aerodynamic behaviour of a standard radar scanner used for maritime applications. The radar scanner is a bluff body more than $2 \mathrm{~m}$ long that, during its operating conditions, rotates under relative wind. The requirements for good radar performances are an almost constant angular velocity under different wind conditions, and the capability to start from rest under strong wind, with a consequent large dimensioning torque and power of the motor. An experimental campaign and a numerical study have been carried out in order to better understand the flow-structure interaction. Experimental tests were performed at Politecnico di Milano wind tunnel on the radar scanner in full scale both in static and in dynamic conditions. During static tests the aerodynamic forces on the radar scanner have been measured changing the wind angle of attack. The dynamic tests have been carried out using the electric motor and the gearbox normally used to move the radar. Critical operating conditions have been reproduced driving the radar to rotate under strong wind velocity and measuring the motor torque and the radar angular velocity. Both the tests pointed out a very high aerodynamic moment opposing the radar rotation, thereby reducing its velocity to point of stopping it. In order to better understand how the radar interacts with the flow, 3D CFD analysis has been carried out comparing the results against the experimental data.
\end{abstract}

Keywords: radar aerodynamic coefficients, wind tunnel tests, CFD analysis.

\section{Introduction}

The dynamic behaviour of radar antennas is strongly affected by the fluidstructure interaction between the relative wind and the bluff body dynamics of 
the mechanism. In maritime applications the problem becomes even more severe due to the high wind velocity at the sea level and the relative boat speed. Since the optimal operating conditions suppose an almost constant rotating speed, in some situations the aerodynamic loads can rise up to harm the radar functionality and even stop it. Furthermore, the aerodynamic loads might prevent the antenna from starting up under strong wind conditions if it was at rest, compromising its functionality.

For these reasons an investigation about the radar aerodynamic behaviour becomes useful, especially at the design stage, allowing for a correct motor dimensioning and an optimization of the body shape.

Bluff bodies are characterized by high aerodynamic forces that can strongly change, modifying the section shape $[1,2]$, and also by important 3D effects, which can influence the overall forces. In particular, the shape of the extremity, ruling the downstream wake, results in different forces [3]. In literature some studies about the rotating antennas can be found. This research activity, attributable to Lombardi $[4,5]$, deals with an experimental investigation of the problem and an optimization stage through the adoption of particular fins on the body back.

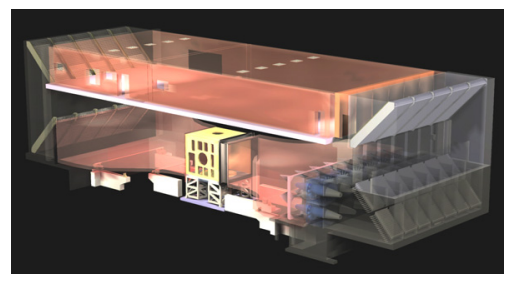

(a)

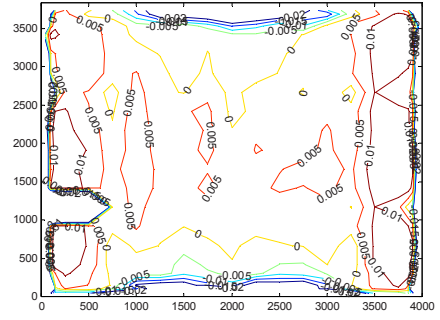

(b)

Figure 1: $\quad$ Politecnico di Milano wind tunnel (a) and wind speed variation with respect to the mean value in the measurement cross-section (b).

In this paper a generic maritime radar shape has been studied. An experimental campaign has been carried out at the Politecnico di Milano wind tunnel. A preliminary static analysis allowed one to define the static aerodynamic coefficients at different exposure angles. Then, the same radar antenna was tested in operating conditions, i.e. reproducing the radar rotation under the effect of different wind speeds. Finally, in order to better investigate the pressure and velocity fields around the body, a 3D CFD analysis was carried out.

\section{The experimental campaign}

The experimental tests have been performed on the low turbulence-high velocity test section of the Politecnico di Milano wind tunnel (Figure 1(a)). 


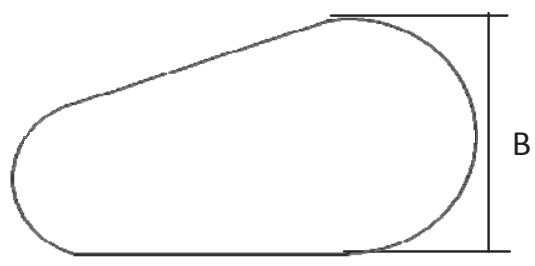

(a)

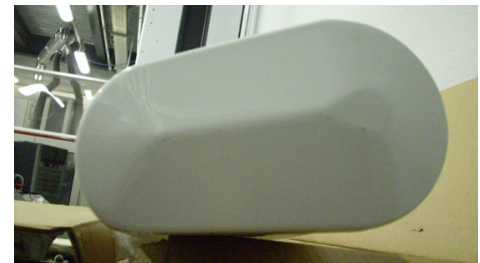

(b)

Figure 2: $\quad$ The radar antenna cross-section (a) and correspondent photo (b).

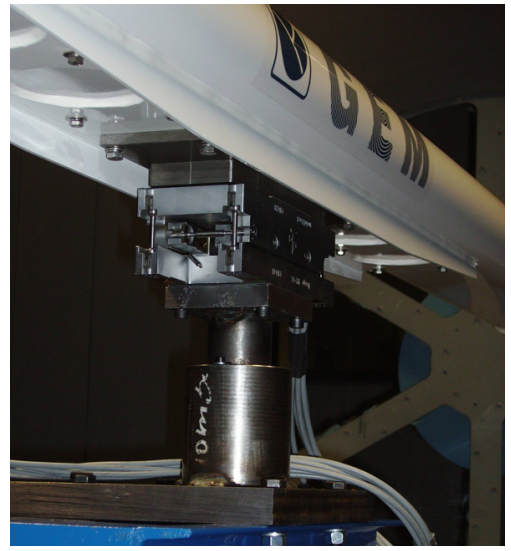

(a)

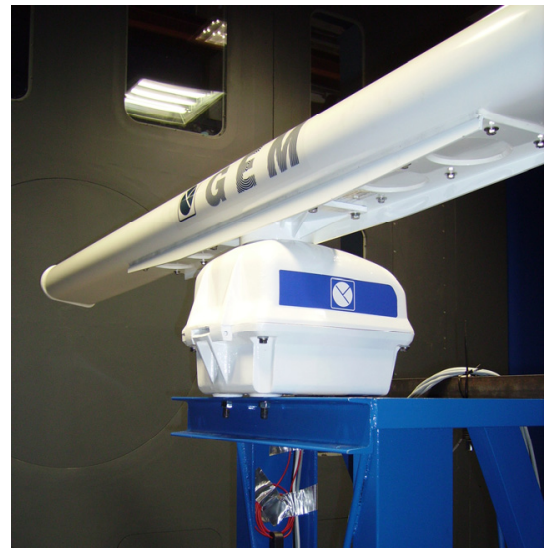

(b)

Figure 3: The antenna experimental set-up: the support and balance for the static tests (a) and the motorized support the dynamic tests (b).

In Figure 1(b) the contour lines of the wind speed variation, with respect to its mean value, are reported on the measurement section. A very uniform distribution can be appreciated, especially in the central region, denoting a strongly smooth flow.

The radar antenna used for the study is $\mathrm{L}=2.200 \mathrm{~m}$ long and it has an asymmetric section reproduced in Figure 2, where $B=0.108 \mathrm{~m}$ represents its characteristic dimension. It was placed in the middle of the test section in order to obtain reduced blockage and wall proximity effects.

Figure 3 shows the two experimental set-ups for static and dynamic tests. During static tests all the aerodynamic forces were measured through a dynamometric balance rigidly connected to the radar and placed on a special device designed to allow one to change the relative angle between the model and the incoming wind.

During the dynamic tests the same antenna was placed directly on the electric motor used in its operating conditions, measuring the torque from the power absorbed by the motor and the angular velocity of the radar. The radar motor is not controlled and it supplies a torque according to its characteristic torque/power curve. 


\section{The experimental results}

In this section the most significant aerodynamic loads measured on the radar antenna in static conditions are reported. Among the six force components, the $\operatorname{drag}\left(\mathrm{F}_{\mathrm{D}}\right)$ and the lateral $\left(\mathrm{F}_{\mathrm{L}}\right)$ forces together with the torsion moment $\left(\mathrm{M}_{\mathrm{z}}\right)$ have been investigated since they are the most significant for the radar dynamic behaviour in the rotation plane. They are expressed according to the sign convention reported in Figure 4(a). The reduction point used for moment definition belongs to the rotation axis, while the positive direction is assumed consistent with the positive direction of the rotation angular velocity.

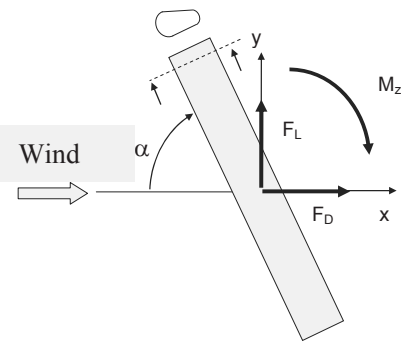

(a)

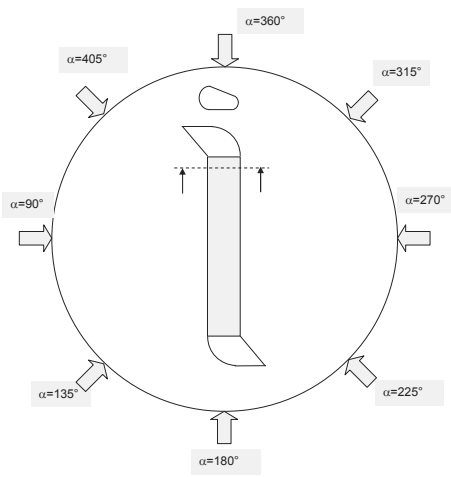

(b)

Figure 4: The reference system used for the forces and moment calculation and measurement (a) and for the wind exposure angles (b).

The results are represented in terms of static aerodynamic coefficients, defined as:

$$
C_{D}=\frac{\bar{F}_{D}}{\bar{q} B L}, \quad C_{L}=\frac{\bar{F}_{L}}{\bar{q} B L}, \quad C_{M z}=\frac{\bar{M}_{Z}}{\bar{q} B^{2} L}
$$

where $\mathrm{q}$ is the mean dynamic pressure blowing on the radar, while the other terms have been previously introduced.

For each wind exposure angle, as described in Figure 4(b), the coefficients have been calculated and the results are reported in Figure 5.

It is possible to notice that the maximum drag was measured in correspondence of $\alpha=90 \mathrm{deg}$ when the radar exposes the larger curvature profile to the incoming wind. Lateral force and moment have similar trend reaching the maximum positive value at $\alpha=220 \mathrm{deg}$ and the maximum negative value at $\alpha=325 \mathrm{deg}$, where negative moment means that it acts against the radar rotation direction, i.e. it is a resistant moment. It is possible to notice that the radar antenna is characterized by negative moment when the smaller curvature profile is directly exposed to the wind so the most critical conditions occur for high values of lateral force, not in correspondence of the maximum value of drag force. 


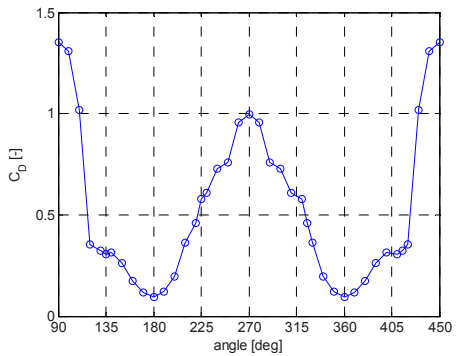

(a)

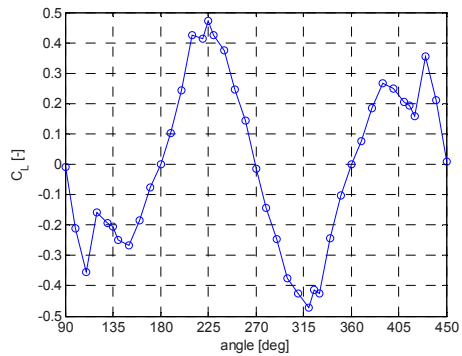

(b)

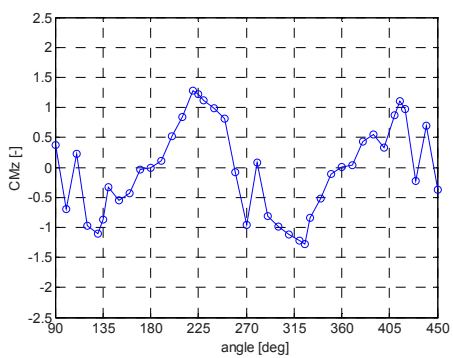

(c)

Figure 5: The experimental tests; the static aerodynamic coefficient as a function of the wind exposure angle; the drag (a) and lateral (b) force coefficient and the torsion moment (c) coefficient.

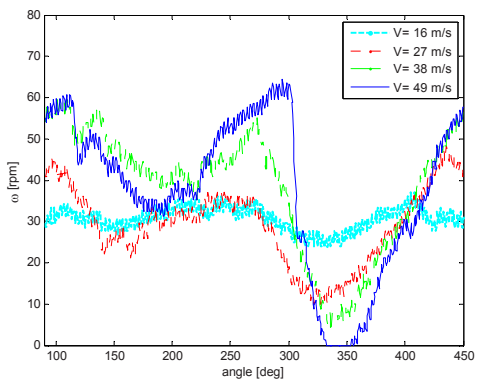

(a)

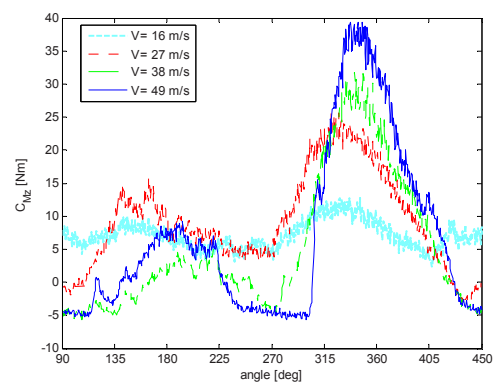

(b)

Figure 6: The experimental tests; the motor angular velocity (a) and resistant torque (b) as a function of the angular position during the dynamic tests.

Figure 6 resumes the dynamic tests results: Figure 6(a) shows the radar angular velocity and Figure 6(b) the motor torque as a function of the angular position. The radar operating conditions become more critical increasing the wind velocity: for high wind velocity, the angular speed is very irregular during 
a cycle reaching low value for $\alpha=300-350 \mathrm{deg}$, the range characterized by high resistant moment during static tests.

The Figure 7 compares static and dynamic results for $\mathrm{V}=38 \mathrm{~m} / \mathrm{s}$ in terms of moment coefficient. The dynamic moment has been obtained by subtracting the inertial contribute of the radar from the motor torque. It is possible to observe a good agreement between the two data. On the other hand, a not negligible effect of unsteady flow condition is present in the dynamic tests, resulting in a more onerous torque request compared to the steady flow conditions.

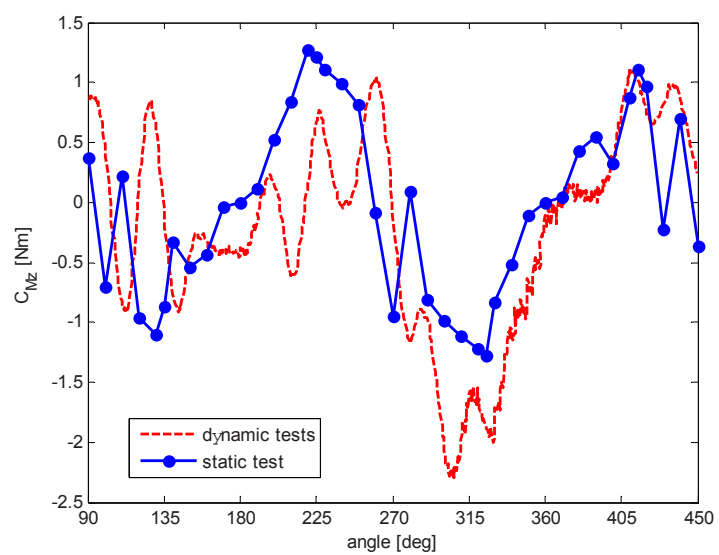

Figure 7: The experimental tests; the aerodynamic torsion moment coefficient; comparison between the static and the dynamic (without inertial contribution) case.

\section{The 3D CFD analysis}

CFD analyses have been performed to investigate the flow field around the radar. In fact, numerical studies allow one to collect more information with respect to the experimental tests on both the static pressures on the object surfaces and the velocity field around it.

The investigation of the flow field around the antenna at $\alpha=315 \mathrm{deg}$, with respect to the wind direction, was performed by means of 3D CFD analyses. The choice of this angle is due to the critical operating conditions previously described in the $\alpha=300-350$ deg range. The 3D simulations have been carried out in order to correctly reproduce the fluid-structure interaction phenomena that cannot be bounded in a plane (2D study).

The considered geometry represents the 1:1 scale model. The simulation results have been validated by a comparison between experimental and numerical results in terms of global coefficients. All the CFD analyses have been performed using the commercial code FLUENT $^{\circledR}$.

The CFD analyses have been performed at a wind speed of $50 \mathrm{~m} / \mathrm{s}$ (the highest speed tested during the experimental campaign). This speed corresponds 
to a Reynolds number equal to $3.70 \cdot 10^{5}$, referred to the radar characteristic dimension. Considering the low turbulence intensity $\left(\mathrm{I}_{\mathrm{u}}=2 \%\right)$ of the wind tunnel flow and the expected low levels of self-induced turbulence, the complete Navier-Stokes equations were solved without any turbulence model. A full direct numerical simulation is not feasible at the considered large Reynolds number because of the amount of computational effort required. Therefore it has been decided to perform an analysis of the problem consisting in solving the NavierStokes equations on a coarser mesh (with respect to the DNS requirements) tolerating that the results will be affected by the approximation of the lack of the contribution of all the small turbulence scale filtered out by the grid spatial dimension. In particular, close to the wall, where the smallest turbulence scales and the largest gradient are present, the adoption of a grid with larger dimensions will result in underestimating the slope of the velocity profile and consequently the estimation of the shear stresses.

In this work the hexahedral cell meshing technique is adopted, with a finer spatial resolution closed to the antenna surface (the minimum cell dimension is set to $0.1 \mathrm{~mm}$ on the surface) in order to correctly reproduce the geometrical details and to better approximate the more severe gradients of this zone. A coarser spatial resolution is kept in the exterior region where the flow is less perturbed. The total cells number is about 2.5 million. The simulations have been performed on a cluster Xeon - Exadron (32 bit, using 3 bi-processor nodes, Intel Xeon $3.06 \mathrm{GHz}$ ) of CILEA.

The analyses were performed considering the flow as uncompressible and using a segregated solver. A first and a second order upwind method were chosen for the Navier-Stokes equations while the velocity-pressure coupling has been performed with a SIMPLE scheme.

A velocity inlet boundary condition is applied on the front plane $(2 \times 10 \mathrm{~m})$ at about $6 \mathrm{~m}$ from the vehicle (Figure 8(a)). A uniform velocity profile is adopted in order to compare the results with the experimental ones where the floor boundary layer is very thin and the wind speed distribution was very uniform.

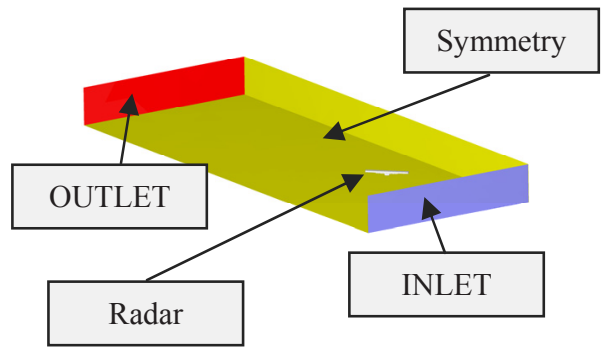

(a)

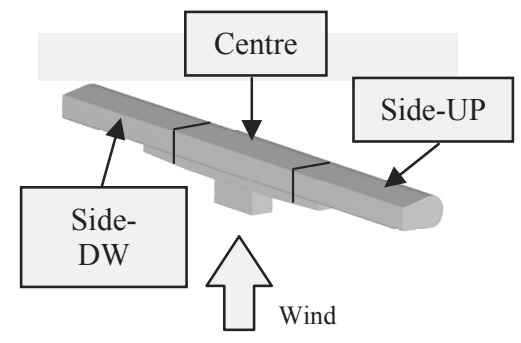

(b)

Figure 8: CFD analysis: the calculation domain (a) and the radar antenna 3D model split into the three main parts (b). 
On the rear plane $(20 \mathrm{~m}$ downwind the model in order to allow a correct evolution of the velocity field) a pressure outlet condition is set to a gauge value equal to 0 . For the other four planes, confining the flow, the symmetry condition is considered.

The geometry of the solid model consists in the radar main body and a connection box to the motor. In Figure 8(b) the CAD 3D model is reported. The whole model is divided into three parts along the radar main direction in order to investigate their contribution to the global coefficients. These three parts are defined in the following as:

- Side-UP: the lateral part upwind in the 315 deg exposure $(33 \%$ of total length L);

- Centre: the central part (34\% of total length L);

- Side-DW: the lateral part downwind in the 315 deg exposure $(33 \%$ of total length L).

The air has been considered in standard conditions: $\mathrm{P}=101325 \mathrm{~Pa}, \mathrm{~T}=288.15$ $\mathrm{K}, \rho=1.225 \mathrm{~kg} / \mathrm{m} 3, \mu=1.789410-5 \mathrm{~kg} /(\mathrm{m} / \mathrm{s})$.

Table 1: $\quad$ CFD analysis; the static aerodynamic coefficients $\left(\mathrm{C}_{\mathrm{D}}, \mathrm{C}_{\mathrm{L}}\right.$ and $\mathrm{C}_{\mathrm{Mz}}$ ) at the wind exposure angle $\alpha=315 \mathrm{deg}$ for the whole model and the three main parts.

\begin{tabular}{|l|c|c|c|}
\hline & $\mathrm{C}_{\mathrm{D}}$ & $\mathrm{C}_{\mathrm{L}}$ & $\mathrm{C}_{\mathrm{Mz}}$ \\
\hline Side-UP & 0.148 & -0.107 & -1.285 \\
\hline Centre & 0.281 & -0.244 & -0.077 \\
\hline Side-DW & 0.084 & -0.019 & 0.440 \\
\hline TOTAL & 0.513 & -0.370 & -0.922 \\
\hline
\end{tabular}

In Table 1 the numerical results are reported. They can be compared with the same coefficients experimentally measured at $\alpha=315 \mathrm{deg}$ (Figure 5). It is possible to notice that the CFD analysis slightly underestimates the three coefficient absolute values. This is attributable to the wall shear stress treatment and the coarsen mesh in the wake region previously described.

The comparison between the three part contributions put in evidence that the force coefficients are mainly due to the radar central part characterized by a larger front surface, while the downwind side contribution is nearly null. The $\mathrm{C}_{\mathrm{Mz}}$ coefficient on the other hand is mainly affected by the side parts and in particular by the upwind one.

In order to better describe this result, in Figure 9 the static pressure distribution on the antenna surfaces is reported together with the flow path lines.

In Figure 9(a) it is possible to notice the positive pressure on the radar front surface that is the main responsible for the $C_{D}$ and $C_{L}$ force coefficients. Moreover it is important to highlight the higher values of pressure in the upwind side, and in particular the stagnation point on the lateral face (the left side in Figure 9(b)). This contribution, although associated to a quite small area, strongly affects the $\mathrm{C}_{\mathrm{Mz}}$ coefficient thanks to the large distance with respect to the moment centre point (rotation axis). 


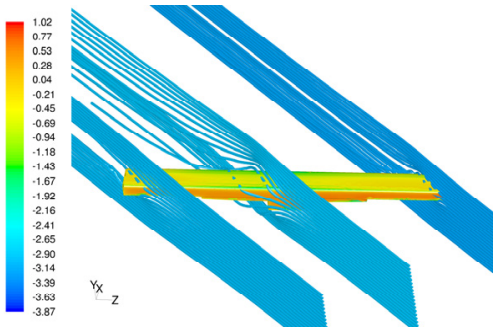

(a)

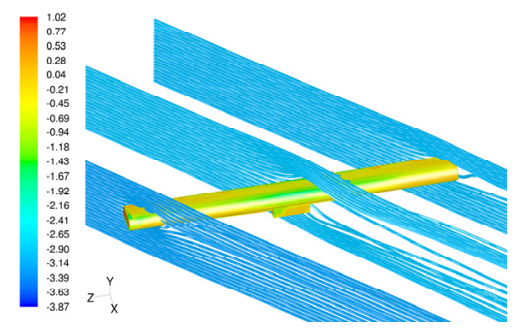

(b)

Figure 9: CFD analysis; the front (a) and rear view (b) of the static pressure distribution on the radar surface and the flow path lines.

\section{Conclusions}

An aerodynamic analysis has been performed on a radar antenna with the goal to define and to improve the performances under strong wind conditions. In the paper a combined use of wind tunnel investigation and CFD study has been adopted. A simple laminar 3D solution succeeded in reproducing the main flow patterns allowing for an interpretation of the experimental results.

The static aerodynamic coefficients are well representative of the system behaviour in unsteady flow (i.e. rotation of the radar). The unsteady effects translate in a worsening of the peak torque required by the motor in the whole 0 360 deg range.

These effects are mainly due to the antenna cross-section asymmetry leading to more severe aerodynamic loads at $315 \mathrm{deg}$ exposure angle. For this reason a first improvement could be obtained adopting a symmetric section.

The worsening effects are also associated by the fluid structure interaction in the radar side parts. The introduction of appendices in correspondence of the antenna tips could reduce the resistant moment, due to the upwind extreme portion, and enhance the push of the analogous downwind part.

\section{Acknowledgements}

The research has been developed with the financial support of GEM ELETTRONICA s.r.l., which also provided the physical model for the wind tunnel experimental tests.

\section{References}

[1] M.M. Zdravkovich, 1997, Flow Around Circular Cylinders - Vol. 1: Fundamentals, Oxford University Press, New York, United States.

[2] E. Simiu, R.H. Scanlan, Wind effects on structures, John Wiley \& Sons, 1996. 
[3] Zasso A., Larose G. L., Giappino S. Muggiasca S., Effects of turbulence intensity and surface roughness on stays of cable stayed bridges, Proceedings of Sixth International Symposium on Cable Dynamics, September 19-22, Charleston, South Carolina.

[4] Lombardi, G., 1991. Wind-tunnel tests on a model antenna rotating in a cross flow. Engineering Structures, 13(4), pp. 345-350.

[5] Lombardi, G., 1989. Wind-tunnel tests on a model antenna with different fin configurations. Engineering Structures, 11(3), pp. 134-138. 